Derartige Unterschiede sind viel zu bedeutend, um als systematische Beobachtungsfehler, etwa als Folge einer Änderung der persönlichen Auffassung einer Bisektion, betrachtet werden zu können. Wie ich mich oft durch direkten Versuch überzeugt habe, ist das Auge jederzeit imstande, solche $\mathrm{Be}-$ träge selbst ohne jede Messung wahrzunehmen.

Auch wäre es als ein erstaunlicher Zufall anzusehen, daß derartige systematische Beobachtungsfehler durch bloße Änderung der zwei Konstanten unserer empirischen Rotationsgleichung in jedem einzelnen Fall so vollständig eliminiert werden könnten.

$\mathrm{Da}$ die in Tabelle 3 und 4 gegebenen empirischen Gleichungen die Beobachtungen hinreichend genau darstellen, mag es für das Folgende erlaubt sein, statt der Abweichungen in den Kurven diejenigen in den Werten der Koeffizienten $a$ und $b$ näher zu diskutieren.

$\mathrm{Zu}$ diesem $\mathrm{Z}$ weke stelle ich in Tabelle 5 diese Werte noch einmal zusammen.

Tabelle 5.
Upsala $\left\{\begin{array}{llc}\text { Ep. d. Beob. } & a & b \\ \text { I } 899.5 & 1.98 & -0.57 \\ \text { I } 900.5 & 2.11 & -0.40 \\ \text { I } 901.5 & 2.09 & -0.79 \\ 1901.7 & 2.06 & -0.70 \\ 1902.5 & 1.973 & -0.560 \\ \text { I903.5 } & 2.036 & -0.251 \\ 1904.5 & 2.075 & -0.271 \\ \text { I905.5 } & 2.039 & -0.245 \\ 1906.3 & 2.010 & -0.294\end{array}\right.$

Zunächst bemerkt man, daß sich die Edinburger Reihe in durchaus befriedigender Weise an die Beobachtungen des Herrn Dunér anschließt. Diese Übereinstimmung zwischen zwei Beobachtungsreihen, von verschiedenen Beobachtern mit verschiedenen Instrumenten angestellt, läßt wohl den berechtigten Schluß zu, daß systematische Beobachtungs- und Instrumentalfehler die Resultate nicht wesentlich beeinflußt haben können.

Untersucht man die zeitlichen Änderungen der Werte $a$ und $b$, so findet man deutliche Spuren einer etwa dreijährigen Periode. Für die Konstante $a$ ergeben sich Maxima für die Epochen r901.0 und 1904.5, und ein dazwischen liegendes Minimum für 1902.7, während $b$ etwas frühere Wendepunkte zeigt, nämlich Maxima etwa bei 1900.5 und

Edinburgh, Royal Observatory, August 1906.
I 904.0 und ein Minimum bei I902.0. Dabei macht sich jedoch ein wesentlicher Unterschied zwischen den beiden Konstanten insofern bemerkbar, als $b$ neben der dreijährigen Schwankung noch eine sehr deutliche stetige Abnahme aufweist, die in den $a$.Werten bis jetzt wenigstens nicht aufgetreten ist. Dies wird besonders deutlich bemerkbar, wenn wir durch geeignete Mittelbildung in der Tabelle 5 die dreijährige Periode eliminieren. In der folgenden Tabelle 6 teile ich derartige Mittelwerte mit, die dadurch erhalten wurden, daß je vier aufeinander folgende Jahreswerte vereinigt worden sind.

\begin{tabular}{ccccc} 
& & \multicolumn{3}{c}{ Täglicher Rotationswinkel } \\
Epoche & $a$ & & Aquator & Pol \\
1901.0 & $\mathbf{2 . 0 3 5}$ & -0.569 & $870^{\circ}$ & $627^{\prime}$ \\
1902.0 & 2.048 & -0.489 & 875 & 666 \\
1903.0 & 2.039 & -0.457 & 872 & 676 \\
I 904.0 & $\mathbf{2 . 0 3 1}$ & -0.332 & 868 & 726 \\
1905.0 & 2.040 & -0.295 & 872 & 759
\end{tabular}

Während die Werte von $a$ eine außerordentliche Konstanz zeigen, ist die Abnahme der Werte von $b$ sehr auffallend. Dieses Resultat ist dahin zu interpretieren, daß die Beobachtungen zur Zeit des Fleckenminimums (rgoI) für hohe Breiten bedeutend kleinere Rotationswinkel ergeben, als zur Zeit energischer Fleckentätigkeit (1905).

Ein sicheres Urteil über die Bedeutung dieses merk. würdigen Resultats ist zur Zeit wohl kaum möglich. Es scheint mir dazu unbedingt nötig, die Beobachtungen mindestens über einen vollen Sonnenfleckzyklus fortzusetzen. Sollte sich erweisen, daß die Werte der Konstanten $a$ und $b$ beim nächsten Minimum mit denen des Jahres I90I übereinstimmen, so wäre damit, nach meiner Ansicht, der solare Ursprung der gefundenen Änderungen außer Frage gestellt, und man könnte sich dann wohl kaum länger der Ansicht verschließen, daß das Rotationsgesetz der Sonne durch die sich auf ihr abspielenden dynamischen Vorgänge merklich beeinflußt wird. Diese Bestätigung bleibt vorerst abzuwarten. Trotzdem glaubte ich mit der Veröffentlichung der bis jetzt erlangten Resultate nicht länger zögern zu sollen, da sie selbst in dieser provisorischen Gestalt geeignet sein dürften, das Interesse der auf diesem Gebiete arbeitenden Fachgenossen zu erregen und zu einer allgemeineren und vertiefteren Erforschung dieser für die Sonnenphysik so ungemein wichtigen Erscheinungen den Anstoß zu geben.

\title{
105853n, Nova (154.1906) Velorum, H 1268.
}

\section{(Harvard College Observatory Circular No. I2I).}

During an examination of photographs taken with the I-inch Cooke lens, a new object was found, by Miss Leavitt, in the constellation Vela. It precedes the star C.P.D. $-53^{\circ} 4300$, magn. I0.0, by about $2^{5}$, and is north $2^{\prime \prime}$. Its position for

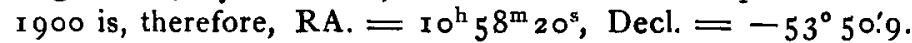
It follows a fifteenth magnitude star by about $2^{\mathbf{s}}$ and is south of it $15^{\prime \prime}$. The Durchmusterung star may be found on the Map of the Sky (Circular 71, A. N. 162.281) in the position
No. 50, [146,101]. Owing to the small scale of these plates, measurements of position and brightness were difficult. The object does not appear on any plate taken before Dec. 5, I905, but is seen on fourteen plates taken since that date. The latest plate taken before its appearance was $\mathrm{AM}_{3718 \text {, }}$ July I 2, I905, on which stars as faint as the magnitude 11.5 are clearly seen. All observations on plates taken since that date and including it, are given in Table $I$, in which the 
successive columns give the plate number, the date, the length of exposure, the Julian Day, and the magnitude. On Plate AM 4 I 43, there are 17 exposures of from 25 to 30 minutes each.

Table I. Observations of the Nova.

\begin{tabular}{|c|c|c|c|c|c|c|c|c|c|c|c|}
\hline Plate & Date & Exp. & J. D. & Magn. & Plate & & Date & & Exp. & J. D. & Magn. \\
\hline $\mathrm{AM}_{3718}$ & 1905 July I2 & $60^{m}$ & $7 \circ 39$ & $<$ II. 5 & $\mathrm{AM}_{4185}$ & 1906 & April & 28 & $60^{m}$ & $73^{29}$ & 10.55 \\
\hline $\mathrm{AM}_{4015}$ & Dec. 5 & 62 & 7185 & $9.7^{2}$ & $\mathrm{AM}_{4194}$ & 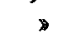 & $\Rightarrow$ & 30 & 60 & 7331 & 10.42 \\
\hline $\mathrm{AM}_{4053}$ & I 906 Jan. 26 & 60 & 7237 & 9.72 & $\mathrm{AM}_{4212}$ & 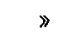 & May & Io & 60 & 7341 & I0.10 \\
\hline $\mathrm{AM}_{4076}$ & $\Rightarrow \quad 30$ & 63 & 7241 & 9.98 & $\mathrm{AM}_{4225}$ & $\gg$ & $>$ & 12 & 60 & 7343 & IO.I 5 \\
\hline $\mathrm{AM}_{409} 8$ & Febr. 3 & 60 & 7245 & 9.95 & $\mathrm{AM}_{4287}$ & 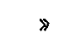 & June & I I & 64 & 7373 & 10.02 \\
\hline $\mathrm{AM}_{4} \mathrm{I} 33$ & Mar. I 7 & 75 & 7287 & 9.80 & $\mathrm{AM} 4300$ & 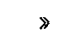 & $\gg$ & I 3 & 65 & 7375 & 9.80 \\
\hline $\mathrm{AM}_{4134}$ & $\gg \quad 21$ & 70 & 7291 & 9.78 & $\mathrm{AM}_{435^{2}}$ & $\searrow$ & $\gg$ & 29 & 60 & 7391 & 11.05 \\
\hline $\mathrm{AM}_{4} \mathrm{I} 43$ & 26 & 485 & 7296 & 9.80 & $\mathrm{AM}_{43} 64$ & 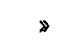 & July & 2 & 60 & 7394 & < II.2 \\
\hline
\end{tabular}

The number of early plates examined, on which the Nova was not seen and was fainter than the eleventh magnitude, was 127 , the numbers taken in successive years being as follows: in 1889 one, 1890 two, $189 \mathrm{I}$ one, 1892 one, I 893 three, 1894 eight, 1895 eleven, 1896 ten, 1897 eighteen, I 898 eleven, 1899 six, I 900 eleven, I 901 ten, 1902 eleven, 1902 seven, 1904 nine, I 905 seven. Two of these plates taken with the 24 -inch Bruce Telescope, and two taken with the 8 -inch Bache Telescope, show stars at least as

faint as the magnitude 15.0 , and twentyfive other plates show stars as faint as the magnitude 13.5 , but no trace of the Nova could be found on any of them.

The Nova shows considerable fluctuation in light during the period covered by the observations, and it is not impossible that it may again become sufficiently bright for its spectrum to be obtained. Even without such proof, there can be little doubt that the object observed is actually a nova.

Harvard College Observatory, 1906 Nov. I 2.

Edward C. Pickering.

\section{Observation d'une blanche tache sur le III. satellite de Jupiter.}

Je me fais un devoir d'appeler tout de suite l'attention des astronomes possédant des puissants instruments, sur la très blanche tache polaire boréale que j'ai observé hier

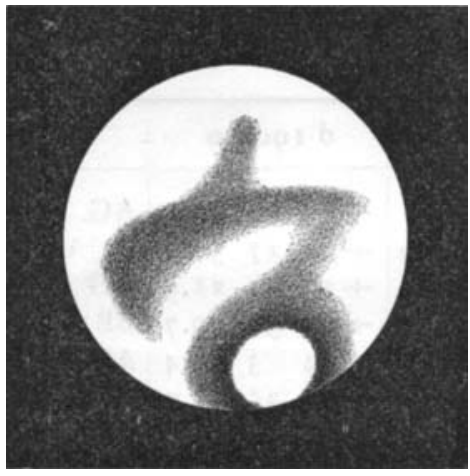

Satellite III de Jupiter. 1906 Nov. $2313^{\mathrm{h}} 30^{\mathrm{m}}$.

Barcelone, le 24 novembre igo6.
23 novembre 1906, sur le troisième satellite de Jupiter avec l'équatorial de $38 \mathrm{~cm}$ de l'Observatoire Fabra.

Elle est d'une éclatante blancheur, bordée d'une région très sombre, à peu près comme on observe dans la planète Mars, et d'une observation relativement facile. Je l'ai observé avec des grossissements de $45^{\circ}$ à $75^{\circ}$ fois. Avec ce dernier grossissement, l'observation de cette tache était d'une facilité extraordinaire. Je crois qu'elle peut être visible avec des instruments bien plus petits.

Le diamètre du satelite passant par le centre de la tache était sensiblement perpendiculaire à la direction des bandes de Jupiter, mais la tache paraissait penchée vers nous. Cet observation est faite entre $13^{\mathrm{h}}$ et $14^{\mathrm{h}}$ I $5^{\mathrm{m}}$. Pendant ce temps, je n'ai observé aucun déplacement de la tache brillante.

On voyait aussi, évidemment, des autres taches sombres dans le reste du disque, mais il etait très difficile de les voir avec quelque précision. L'image télescopique était très belle. Dans son ensemble, le satellite III paraissait une image réduite de la planète Mars.

Fosé Comas Solá, Directeur de l'Observatoire Fabra.

\section{Beobachtungen von kleinen Planeten.}

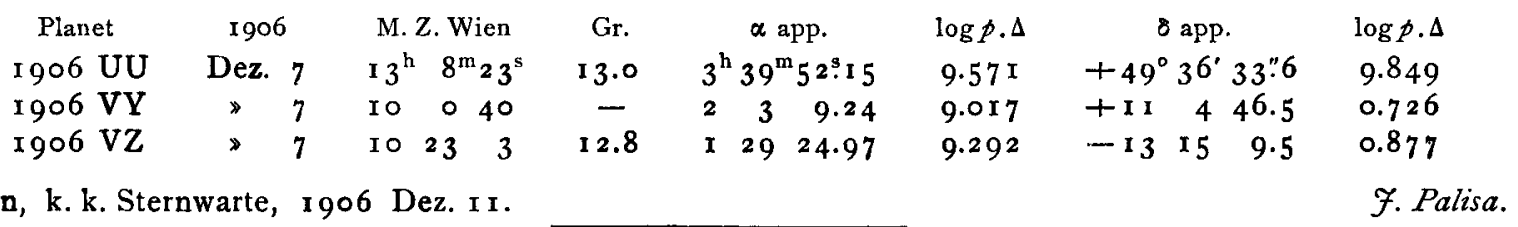

